\title{
Lageroptimierung - ein Praxisbeispiel aus der Möbelbranche
}

\author{
Justyna Zybert \\ Dieser Beitrag wurde auf Basis der Diplomarbeit der Autorin verfasst und gilt als Ergebnis eines von mehreren Projekten in der \\ Möbelbranche in Polen, die von der Autorin im Zusammenhang mit ihrer Diplomarbeit realisiert wurden.
}

\section{Firmenbeschreibung}

Die Möbelfabrik „Wiza“ hat ihren Sitz in Kostrzyn (Wlkp). Die Firma produziert Massivholzmöbel, die zu den besten ihrer Klasse gehören. Die Qualität dieser Möbel wurde bereits mehrfach auf Messen und Ausstellungen ausgezeichnet. Der wichtigste Absatzmarkt für die Erzeugnisse ist Deutschland.

Die Firma bietet ihren Kunden ein breites Sortiment an auch mit der Möglichkeit, das Material, die Farbe, die Holzart usw. individuell auszuwählen. Dieses umfangreiche Angebot sichert der Firma eine gute Marktposition, schafft aber auch die Notwendigkeit, sehr große Materialvorräte zu halten, die hohe Materialbewirtschaftungskosten verursachen.

Die Verkaufsanalyse hat gezeigt, dass ca. 20 Sortimentspositionen nur sehr schlecht verkauft werden. Es gibt keine Notwendigkeit, sie weiter zu produzieren.

Die Fabrik hat drei Standorte: Schreinerei und Polsterung befinden sich an einem Standort, $3 \mathrm{~km}$ weiter hat die Näherei ihren Sitz und 6 km weiter sind Buchhaltung und Personalabteilung angesiedelt. Diese geografische Aufteilung ist sehr ungünstig und verursacht zusätzliche Transport- sowie Kommunikationskosten.

\section{Materialwirtschaft}

Die Materialbewirtschaftung ist in der Firma nicht stark ausgeprägt. Es gibt kein gesondertes Materiallager und Materialausgaben oder Verbräuche werden nicht überwacht. Es werden zu viele Materialen und Produktionsmittel verbraucht. Dies verursacht hohe Materialvorräte mit entsprechend hohen Kosten. Durch Mangel in der Materialerfassung und in der Produktionsplanung werden die Materialbestellungen nicht rationell durchgefuihrt. Im Materiallager herrscht Unordnung. Es gibt keine feste Einteilung in Kommissionierzone, Wareneingang und Warenausgang.

\section{Lager}

Das Lagergebäude und das Fertigteilelager bieten noch freie Kapazitäten. Das Lagerungssystem ist dreidimensional aufgebaut. Die Ein- und Auslagerung in die Lagerregale werden von Gabelstaplern realisiert. Das Lager besitzt eine ausreichende Beleuchtung. Wasseranschliusse oder eine Heizung sind dagegen nicht vorhanden. Im Fertigteilelager werden neben den Fertigerzeugnissen auch Roherzeugnisse gelagert.
Die Analyse der Materiallieferungen hat ergeben, dass zwei Gruppen zu unterscheiden sind. Bei der ersten Gruppe ist der Materialfluss unregelmäßig, aber es gibt nur wenig Wochen, in denen keine Lieferung erfolgt. Bei der zweiten Gruppe gibt es dagegen häufiger Wochen, in denen keine Lieferung erfolgt. Deshalb werden zwei Bestellsysteme vorgeschlagen. Für die erste Gruppe ist die Bestellung nach MRP-Regeln vorgesehen. Wenn es viele kleine Ausgaben gibt, kann das System auf Basis des Meldebestands angewandt werden (Abb. 1).

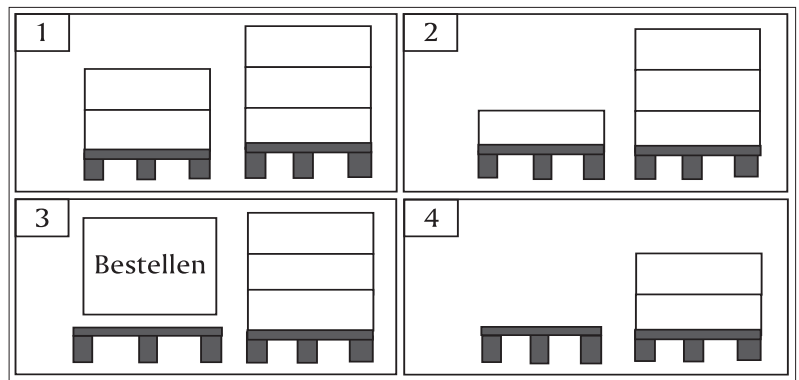

Abb. 1

Die beste Lösung für die zweite Gruppe ist das so genannte „ein für ein“-Bestellsystem. Dieses ist in der Abbildung 2 dargestellt.

\begin{tabular}{|r|r|}
\hline \multicolumn{2}{|c|}{ Bestellung nach dem ,ein für ein“-System } \\
\hline & Ausgabe \\
\hline & Bestellung \\
\hline & \\
\hline & Lieferung \\
\hline
\end{tabular}

Abb. 2: Bestellsystem

\section{Fluss der Bestände: Von der Anlieferung, durch die Annahme und Lagerung bis zur Kommissionierung}

\section{Warenannahme}

Lieferungsdokumente, sowohl aus Deutschland als auch aus Polen, sind Faktura und Frachtbrief. Sowohl im Lager als auch im ganzen Unternehmen gibt es keine standardisierten Abläufe. Die einzelnen Vorgänge werden den Kundenbestellungen zugeordnet.

Einlagerung

Die Lagerplätze werden variabel vergeben. 


\section{Auslagerung}

Der Lagerverwalter identifiziert während der Kommissionierung optisch den Lagerplatz der Ware. Dieser Suchvorgang ist sehr zeitaufwendig und verursacht viele Fehler.

\section{Warenausgang}

Der Warenausgang behandelt nur Fertigerzeugnisse und Komponenten für die Unterlieferanten.

\section{Identifizierte Fehler in der Materialwirtschaft}

- keine internen Dokumente über den Materialfluss

- kein eindeutiges Informationsumlaufsystem

- keine Standardisierung der einzelnen Tätigkeiten

- keine Kompetenzaufteilung im Materialhandling, angefangen bei deren Annahme bis zur endgültigen Abrechnung nach Ablauf des Produktionszyklus

- Defizite bei den Spezifikationen verursachten Desinformation

- fehlende Produktionszeitpläne

- fehlende Materialbedarfsplanung

\section{Projekt des Lagerwirtschaftssystems - Empfehlungen}

\section{Methodik der Lageroperationen}

Materialbewirtschaftung und Lagermanagement sind zwingend zu ändern. Eine effektive Lagerung soll die Lagerfläche maximal ausnutzen und die Anzahl der Einund Auslagerungen minimieren.

Beim Entwerfen der Lagerflächen sind bestimmte Vorgaben zu berücksichtigen. Die Lagerungszone soll am größten sein, die Kommissionierungszone kann mit der Ein- und Auslagerungszone zusammengeschlossen sein, die Warenannahme und der Warenausgang sollen sich in der gleichen Zone befinden.

Besonders wichtig ist die Wahl des Lagerortes und des Lagerplatzes. Hier können drei Methoden Anwendung finden:

1. Methode der freien Lagerungsstellen

2. Methode der fixen Lagerungsstellen

3. Mischmethode (freie und fixe Lagerungsstellen)

Im Falle des beschriebenen Lagers bietet sich die dritte Lösung an. Der Lagerungsprozess soll hier drei Phasen umfassen:

- Wareneingang

- Kommissionierung

- Warenausgang

In jeder Phase soll ein für die einzelnen Operationen verantwortlicher Lagermitarbeiter eingesetzt werden. Die Prozesse sind zu kontrollieren und eventuelle Fehler schnell zu beheben.

Sehr wichtig ist die richtige Unterbringung im Regal. Dazu werden im Lager die Lagerplätze gemäß der Mischmethode (freie und fixe Lagerplätze) vergeben.

Die Kommissionierung wird durch die dynamische Methode „Mann zu Ware“ realisiert. Vorräte sind aus dem Lager zu entnehmen und mit speziellen Lagergeräten zur
Kommissionierungszone zu transportieren. Dort wird die bestellte Lieferung kommissioniert.

Die Kommissionierung ist leichter, wenn es im Lager ein Adresssystem gibt. In der Möbelfabrik „Wiza“ wurden automatische Identifikationssysteme eingefuihrt. Jeder Lagerplatz hat ein eigenes Adressetikett, das mit einem Strichcode versehen ist. Dieser Code ist im IT-System erfasst. Das System zeigt auf einer Übersichtskarte, wo die Ware liegt. Das IT-System erstellt aber auch herkömmliche Dokumente, wie z. B. Belege oder Faktura.

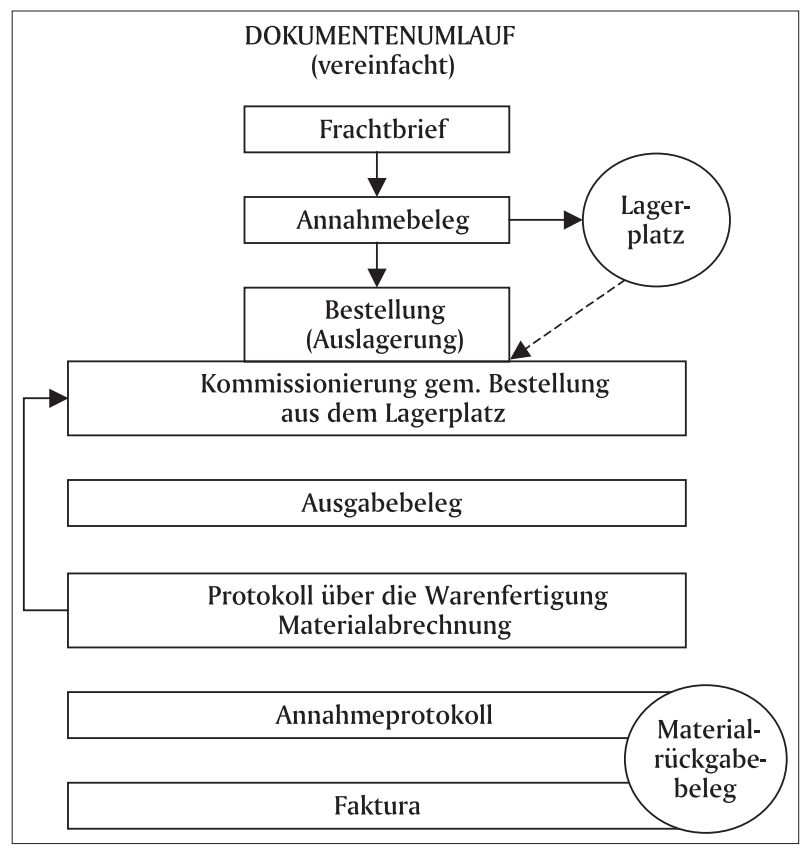

Abb. 3 Vorschlag des Dokumentenumlaufs

Richtige Material- und Lagerwirtschaft bringt berechenbare Effekte. Diese umfassen z. B.

- Senkung der Vorratskosten,

- maximale Ausnutzung der Lagerungsfläche,

- hohe Qualität.

Um diese Effekte zu erreichen, müissen laufende Prozesse ständig verbessert und innovative Informationsverarbeitungssysteme aufgebaut werden.

Die Möbelfabrik „Wiza“ hat die vorgeschlagenen neuen Managementregeln eingesetzt. Dazu mussten Abteilungen reorganisiert und viele Prozesse standardisiert werden. So konnte auch die Material- und Lagerwirtschaft reorganisiert werden. Dort wurden Kosteneinsparungen (Materialkosten, Bestandskosten) und eine bessere Flächenausnutzung realisiert. Diese Ergebnisse tragen direkt zu einer Verbesserung der Wettbewerbssituation der Möbelfabrik „Wiza“ bei.

\section{Autorin}

Justyna Zybert

Studentin der Fachhochschule fuir Logistik Poznan

ul. Estkowskiego 6, 61-755 Poznan, Polen

Tel. +48618529555, +48618510604

E-Mail:wsl@dziekanat.wsl.com.pl 